\title{
Properties and Applications of a New Extended Gamma Function Involving Confluent Hypergeometric Function
}

\author{
Abdus Saboor $\mathbb{D}^{1},{ }^{1}$ Gauhar Rahman $\mathbb{D}^{2},{ }^{2}$ Hazrat Ali, ${ }^{1}$ Kottakkaran Sooppy Nisar (D), \\ and Thabet Abdeljawad $\mathbb{i D}^{4,5,6}$ \\ ${ }^{1}$ Institute of Numerical Sciences, Kohat University of Science and Technology, Kohat 26000, Pakistan \\ ${ }^{2}$ Department of Mathematics and Statistics, Hazara University, Mansehra, Pakistan, Pakistan \\ ${ }^{3}$ Department of Mathematics, College of Arts and Sciences, Prince Sattam Bin Abdulaziz University, Wadi Aldawser 11991, \\ Saudi Arabia \\ ${ }^{4}$ Department of Mathematics and General Sciences, Prince Sultan University, P. O. Box 66833, Riyadh 11586, Saudi Arabia \\ ${ }^{5}$ Department of Medical Research, China Medical University, Taichung 40402, Taiwan \\ ${ }^{6}$ Department of Computer Science and Information Engineering, Asia University, Taichung 40402, Taiwan
}

Correspondence should be addressed to Thabet Abdeljawad; tabdeljawad@psu.edu.sa

Received 4 August 2020; Revised 5 January 2021; Accepted 29 January 2021; Published 25 February 2021

Academic Editor: Antonio Di Crescenzo

Copyright $\left({ }_{0} 2021\right.$ Abdus Saboor et al. This is an open access article distributed under the Creative Commons Attribution License, which permits unrestricted use, distribution, and reproduction in any medium, provided the original work is properly cited.

In this paper, a new confluent hypergeometric gamma function and an associated confluent hypergeometric Pochhammer symbol are introduced. We discuss some properties, for instance, their different integral representations, derivative formulas, and generating function relations. Different special cases are also considered.

\section{Introduction}

The classical gamma function was first found by Swiss mathematician Leonhard Euler (1707-1783) in his objective to generalize the factorial to nonintegral values. The classical gamma function and its related Pochhammer symbol are characterized as follows:

$$
\Gamma(z)=\int_{0}^{\infty} t^{z-1} e^{-t} \mathrm{~d} t, \quad \mathfrak{R}(z)>0
$$

and

$$
(z)_{\rho}= \begin{cases}\frac{\Gamma(z+\rho)}{\Gamma(z)}, & \\ z(z+1) \ldots(z+n-1),\left(\rho=n \in \mathbb{N}_{0}\right), & z \in \mathbb{C}, \\ 1, & (\rho=0, z \in \mathbb{C} \backslash\{0\}) .\end{cases}
$$

The gamma function belongs to category of the special transcendental functions. It has applications in different fields, e.g., definite integration, asymptotic series, hypergeometric series, etc. In the last three decades, various extended forms of classical gamma functions are introduced by different researchers and mathematicians. Among those, 
some of the extended gamma functions and their related Pochhammer symbols are defined below.

Chaudhry and Zubair [1] introduced an extended gamma function, defined by

$$
\Gamma_{\kappa}(z)=\int_{0}^{\infty} t^{z-1} e^{-t-k / t} \mathrm{~d} t, \quad \Re(\kappa)>0, \Re(z)>0
$$

Srivastava et al. [2] proposed the following extended form of Pochhammer symbol:

$$
(z ; \kappa)_{\rho}= \begin{cases}\frac{\Gamma_{\kappa}(z+\rho)}{\Gamma(z)}, & \Re(\kappa)>0, z, \rho \in \mathbb{C}, \\ (z)_{\rho}, & \kappa=0, z, \rho \in \mathbb{C} \backslash\{0\} .\end{cases}
$$

Srivastava et al. [3] introduced an extended gamma function involving modified Bessel function and its associated Pochhammer symbol, defined as follows:

$$
\Gamma_{v}(z ; \kappa)=\sqrt{\frac{2 \kappa}{\pi}} \int_{0}^{\infty} t^{z-3 / 2} e^{-t} K_{v+1 / 2}\left(\frac{\kappa}{t}\right) \mathrm{d} t
$$

and

$$
(z ; \kappa, v)_{\rho}= \begin{cases}\frac{\Gamma_{v}(z+\rho ; \kappa)}{\Gamma(z)}, & \Re(\kappa)>0, \mathfrak{R}(v)>0, z, \rho \in \mathbb{C}, \\ (z ; \kappa)_{\rho}, & v=0, z, \rho \in \mathbb{C} \backslash\{0\},\end{cases}
$$

where $\mathfrak{R}(\kappa)>0, \mathfrak{R}(z)>0$ and $K_{\nu}($.$) is the modified Bessel$ function of order $v$ defined by

$$
K_{v}(y)=\sum_{i=0}^{\infty} \frac{(-1)^{i}(y / 2)^{n+2 i}}{i ! \Gamma(n+i+1)} .
$$

Safdar et al. [4] defined an extension of classical gamma function and its associated Pochhammer symbol involving Mittag-Leffler function, defined by

$$
\Gamma_{s}(z, \sigma)=\Gamma_{s}^{\sigma}(z)=\int_{0}^{\infty} t^{z-1} e^{-t} E_{\sigma}\left(\frac{-s}{t}\right) \mathrm{d} t
$$

and

$$
(z ; s, \sigma)_{\rho}= \begin{cases}\frac{\Gamma_{s}(z+\rho ; \sigma)}{\Gamma(z)}, & \Re(s)>0, \mathfrak{R}(\sigma)>0, z, \rho \in \mathbb{C}, \\ (z ; s)_{\rho}, & \sigma=1, z, \rho \in \mathbb{C} \backslash\{0\},\end{cases}
$$

where $\mathfrak{R}(\rho)>0, \mathfrak{R}(z)>0$, and $E_{\sigma}(z)$ is the Mittag-Leffler function defined by

$$
E_{\sigma}(z)=\sum_{j=0}^{\infty} \frac{z^{j}}{\Gamma(j \sigma+1)}
$$

Srivastava et al. [5] present the familiar incomplete gamma functions $\gamma(s, x)$ and $\Gamma(s, x)$, and they introduce the incomplete Pochhammer symbols that led us to a natural generalization and decomposition of a class of hypergeometric and other related functions which are potentially useful in closed-form representations of definite and semiinfinite integrals of various special functions. Applications of these functions can be found in communication theory, probability distributions, and groundwater pumping modelling.

Şahin et al. [6] introduce a new generalization of the Pochhammer symbol by means of the generalization of extended gamma function as follows:

$$
\Gamma_{p, q}^{\kappa, \mu}(z)=\int_{0}^{\infty} t^{z-1} \exp \left(\frac{-t^{\kappa}}{p}-\frac{q}{t^{\mu}}\right) \mathrm{d} t .
$$

Using the generalization of Pochhammer symbol, they give a generalization of the extended hypergeometric functions of one or several variables. Also, they obtain various integral representations, derivative formulas, and certain properties of these functions.

The rest of the paper is organized as follows. In Section 2, we define a confluent hypergeometric gamma (CHG) function and derive its closed form in terms of Meijer's $G$-function, which is built in function of Computational Package Mathematica. In Section 3, a confluent hypergeometric Pochhammer (CHP) symbol is defined and some of its associate properties are also derived. In Section 4, we define an extension of generalized hypergeometric gamma (GHG) function and derive some of its associate properties. In Section 5, we obtain families of generating function relations. In Section 6, we present comparison of confluent hypergeometric gamma $(\mathrm{CHG})$ function with classical and extended gamma functions for certain numerical values using Table 1. Comparison of integral and closed form of confluent hypergeometric gamma ( $\mathrm{CHG}$ ) function is shown in Table 2. Concluding remarks are given in Section 7 .

\section{A Confluent Hypergeometric Gamma Function}

We propose further generalization of extended gamma function (3) by inducting a confluent hypergeometric function [7] in the integrand of the integral. In Theorem 1 , we have derived the closed-form representation of the proposed CHG function using the inverse Mellin transform technique [8] in the form of Meijer's G-function. 
TABLE 1: Comparison between gamma, extended gamma (3), and CHG (12) functions.

\begin{tabular}{lccc}
\hline$z$ & $\Gamma(z)$ & $\Gamma_{p}(z)$ & $\Gamma_{p}(z ; m, b, \alpha)$ \\
\hline 0.5 & 1.77245 & 0.09769 & 0.02519 \\
1 & 1 & 0.13152 & 0.02863 \\
1.5 & 0.88627 & 0.19042 & 0.03455 \\
2 & 1 & 0.29568 & 0.04426 \\
2.5 & 1.32934 & 0.49078 & 0.06013 \\
3 & 2 & 0.86756 & 0.08654 \\
3.5 & 3.32335 & 1.62685 & 0.13177 \\
4 & 6 & 3.22362 & 0.21197 \\
4.5 & 11.6317 & 6.72464 & 0.35966 \\
5 & 24 & 14.7164 & 0.64269 \\
\hline
\end{tabular}

TABLE 2: Comparison between integral form of CHG function and its closed form.

\begin{tabular}{lcc}
\hline$z$ & Integral form & Closed form \\
\hline 0.5 & 0.02519 & 0.02519 \\
1 & 0.02863 & 0.02863 \\
1.5 & 0.03455 & 0.03455 \\
2 & 0.04426 & 0.04426 \\
2.5 & 0.06013 & 0.06013 \\
3 & 0.08654 & 0.08654 \\
3.5 & 0.13177 & 0.13177 \\
4 & 0.21197 & 0.21197 \\
4.5 & 0.35966 & 0.35966 \\
5 & 0.64269 & 0.64269 \\
\hline
\end{tabular}

Theorem 1. One has the following closed form for $\Gamma_{p}(z ; m, b, \alpha)$.

$$
\begin{aligned}
\Gamma_{p}(z ; m, b, \alpha) & =\int_{0}^{\infty} t^{z-1} e^{-t-p / t}{ }_{1} F_{1}(m ; b,-\alpha t) \mathrm{d} t \\
& =\frac{\Gamma(b)}{\Gamma(m)} \sum_{n=0}^{\infty} \frac{p^{n}}{n !}\left[\begin{array}{cc}
\left.G_{3,3}^{2,2}\left(\begin{array}{c}
1-m, 1-z, 1-z+n \\
0,1-z, 1-b
\end{array}\right)-p^{z} G_{4,2}^{2,2}\left(\begin{array}{cc}
p \alpha \mid & 1-m, 1-z \\
0,1-z, 1-b,-z-n
\end{array}\right)\right]
\end{array}\right.
\end{aligned}
$$

where $\Re(p)>0,|\arg (\alpha t)|<\pi / 2, \mathfrak{R}(z) \neq 0,-1,-2, \ldots$ and $\Re(b) \neq 0,-1,-2, \ldots$

Proof. From [Rainville [7], p. 102, Theorem 36], (12) becomes

$$
\begin{aligned}
\Gamma_{p}(z ; m, b, \alpha) & =\int_{0}^{\infty} t^{z-1} e^{-t-p / t} \frac{1}{2 \pi i} \int_{c-i \infty}^{c+i \infty} \frac{(\alpha t)^{s} \Gamma(-s) \Gamma(m+s)}{\Gamma(b+s)} \mathrm{d} s \mathrm{~d} t \\
& =\frac{\Gamma(b)}{\Gamma(m)} 2(p)^{z / 2} \frac{1}{2 \pi i} \int_{c-i \infty}^{c+i \infty}\left(\frac{\sqrt{p}}{\alpha}\right)^{-s} \frac{\Gamma(-s) \Gamma(\lambda+s)}{(b+s)} k_{z+s}(2 \sqrt{p}) \mathrm{d} s
\end{aligned}
$$

where $k_{v}$ is Macdonald function [9], defined by

$$
k_{v}=\frac{\pi}{2} \frac{I_{-v}(x)-I_{v}(x)}{\operatorname{Sin} v \pi},
$$

and $I_{v}(x)$ is a modified Bessel function of first kind [10], defined by

$$
I_{v}(x)=\sum_{n=0}^{\infty} \frac{(x / 2)^{v+2 n}}{n ! \Gamma(v+n+1)} .
$$


Using (15) and (16) and the residue theorem [11], one has

$$
\begin{aligned}
\Gamma_{p}(z ; m, b, \alpha)= & \frac{\Gamma(b)}{\Gamma(m)} \sum_{n=0}^{\infty} \frac{(p)^{n}}{n !} \\
& \left.\times\left(\begin{array}{l|l}
G_{3,3}^{2,2}\left(\alpha \mid \begin{array}{l}
1, z, b \\
m, z, z-n
\end{array}\right)-p^{z} G_{4,2}^{2,2}\left(\left.\frac{1}{p \alpha}\right|_{m, z} ^{1, z, b, z+n+1}\right.
\end{array}\right)\right),
\end{aligned}
$$

which can be reexpressed as

$$
\Gamma_{p}(z ; m, b, \alpha)=\frac{\Gamma(b)}{\Gamma(m)} \sum_{n=0}^{\infty} \frac{p^{n}}{n !}\left(G_{3,3}^{2,2}\left(\begin{array}{l}
\alpha \mid \begin{array}{c}
1-z, 1-m,-z+n+1 \\
0,1-z, 1-b
\end{array}
\end{array}\right)-p^{z} G_{4,2}^{2,2}\left(\begin{array}{l|l}
p \alpha & \begin{array}{l}
1-z, 1-m \\
0,1-z, 1-b,-z-n
\end{array}
\end{array}\right)\right)
$$

where $\mathfrak{R}(p)>0$ and $\mathfrak{R}(b), \mathfrak{R}(m), \mathfrak{R}(z) \neq 0,-1,-2, \ldots$

Remark 1. It is also important to mention here that in most of the extended cases, no closed form is provided but only new parameter in its function is inducted in the integrand of the integral of classical gamma function or its any extended gamma forms. Moreover, the proposed CHG function may find applications in statistics and a few associations with other special functions and polynomials.

Remark 2. Now, let $m=b$ and $\alpha=0$; then, (12) reduces to extended gamma function defined by Zubair and Chaudhry [12], which is defined as

$$
\Gamma_{p}(z)=\int_{0}^{\infty} t^{z-1} e^{-t-p / t} \mathrm{~d} t
$$

If we consider $p=0$, then (19) becomes the classical gamma function (1).

2.1. Incomplete Confluent Hypergeometric Gamma Functions. Incomplete CHG functions are defined by

$$
\gamma_{p}(z, y ; m, b, \alpha)=\int_{0}^{y} t^{z-1} e^{-t-p / t} F_{1}(m ; b,-\alpha t) \mathrm{d} t
$$

and

$$
\Gamma_{p}(z, y ; m, b, \alpha)=\int_{y}^{0} t^{z-1} e^{-t-p / t}{ }_{1} F_{1}(m ; b,-\alpha t) \mathrm{d} t
$$

Now if we consider $m=b$ and $\alpha=0$, then (20) and (21) are reduced to incomplete gamma function defined by Chaudhry and Zubair [12].

\section{A Confluent Hypergeometric Pochhammer Symbol}

The classical Pochhammer symbol is defined by

$$
(z)_{\rho}=\left\{\begin{array}{l}
\frac{\Gamma(z+\rho)}{\Gamma(z)} \\
z(z+1) \ldots(z+n-1),\left(\rho=n \in \mathbb{N}_{0}\right), \quad z \in \mathbb{C}, \\
1, \quad(\rho=0, z \in \mathbb{C} \backslash\{0\}) .
\end{array}\right.
$$

Pochhammer symbol introduced by Srivastava et al. [2] is given by

$$
(z ; \kappa)_{\rho}= \begin{cases}\frac{\Gamma_{\kappa}(z+\rho)}{\Gamma(z)}, & \Re(\kappa)>0, z, \rho \in \mathbb{C} \\ (z)_{\rho}, & \kappa=0, z, \rho \in \mathbb{C} \backslash\{0\} .\end{cases}
$$

We propose a CHP symbol in following form:

$$
(z ; m, b, \alpha, p)_{\rho}= \begin{cases}\frac{\Gamma_{p}(z+\rho ; m, b, \alpha)}{\Gamma(z)}, & \Re(m, b, \alpha)>0, \mathfrak{R}(p)>0, z, \rho \in \mathbb{C}, \\ (z ; m, b, \alpha)_{\rho}, & p=0, z, \rho \in \mathbb{C} \backslash\{0\},\end{cases}
$$

where $\Gamma_{p}(z ; m, b, \alpha)$ is defined in (12). 
3.1. Some Results Associated with the Confluent Hypergeometric Pochhammer Symbol. Here, we present an integral representation of $\mathrm{CHP}$ symbol $(z ; m, b, \alpha, p)_{\rho}$. Using Equation (12), we define an integral representation of $\Gamma_{p}(z+$ $\rho ; m, b, \alpha)$ as

$$
\Gamma_{p}(z+\rho ; m, b, \alpha)=\int_{0}^{\infty} t^{z+\rho-1} e^{-t-p / t}{ }_{1} F_{1}(m ; b,-\alpha t) \mathrm{d} t .
$$

Combining (25) with (24), one gets an integral representation of $(z ; m, b, \alpha, p)_{\rho}$ in the following form:

$$
(z ; m, b, \alpha, p)_{\rho}=\frac{1}{\Gamma(z)} \int_{0}^{\infty} t^{z+\rho-1} e^{-t-p / t}{ }_{1} F_{1}(m, b,-\alpha t) \mathrm{d} t .
$$

Remark 3. Let $m=b, \alpha=0$, and $p=\kappa$; we get the following Pochhammer symbol defined by Srivastava et al. [2]:

$$
(z ; \kappa, 0)_{\rho}=\frac{1}{\Gamma(z)} \int_{0}^{\infty} t^{z+\rho-1} e^{-t-k / t} \mathrm{~d} t
$$

Theorem 2. One obtains the following result for confluent hypergeometric gamma (CHG) function (12).

$$
\Gamma_{p}(z ; m, b, \alpha)=2 \frac{\Gamma(b)}{\Gamma(m) \Gamma(b-m)} p^{z / 2} \int_{0}^{1} \mu^{m-1}(1-\mu)^{b-m-1}(1+\alpha \mu)^{-z / 2} \times K_{-z}\left(2 \frac{\sqrt{1 / \mu+\alpha}}{\sqrt{1 / p \mu}}\right) \mathrm{d} \mu .
$$

Proof. Using integral form of confluent hypergeometric function, (12) becomes

$$
\Gamma_{p}(z ; m, b, \alpha)=\frac{\Gamma(b)}{\Gamma(m) \Gamma(b-m)} \int_{0}^{\infty} t^{z-1} e^{-t-p / t} \int_{0}^{1} u^{\lambda-1}(1-u)^{b-\lambda-1} e^{-\alpha t u} \mathrm{~d} u \mathrm{~d} t
$$

Apply a one-one transformation but without including the boundaries. Let $v=u t, \mu=t$ in the above equality and the Jacobian is $J=1 / \mu$; then, one gets

$$
\Gamma_{p}(z ; m, b, \alpha)=\frac{\Gamma(b)}{\Gamma(m) \Gamma(b-m)} \int_{0}^{\infty} \int_{0}^{1} \nu^{z-1} \mathrm{e}^{(-v / \mu-\alpha v-p \mu / v)} \mu^{m-z-1} \times(1-\mu)^{b-m-1} \mathrm{~d} \mu \mathrm{d} \nu .
$$

Interchanging the order of integration due to uniform convergence of the integrals and using some basic calculus, one obtains (28).
Theorem 3. For product of two confluent hypergeometric gamma functions, one has the following result:

$$
\begin{aligned}
\Gamma_{p}(z ; m, b, \alpha) \Gamma_{p}(n ; m, b, \alpha)= & 4 \int_{0}^{\pi / 2} \int_{0}^{\infty} \gamma^{2(z+n)-1} \cos ^{2 z-1} \theta \sin ^{2 n-1} \theta \times \exp \left(-\gamma^{2}-\frac{p}{\gamma^{2} \cos ^{2} \theta \sin ^{2} \theta}\right) \\
& \times{ }_{1} F_{1}\left(m ; b ;-\alpha \gamma^{2} \cos ^{2} \theta\right) \times{ }_{1} F_{1}\left(m ; b ;-\alpha \gamma^{2} \sin ^{2} \theta\right) \mathrm{d} \gamma \mathrm{d} \theta .
\end{aligned}
$$

Proof. Substituting $t=\sigma^{2}$ in (12), one gets 
$\Gamma_{p}(z ; m, b, \alpha)=2 \int_{0}^{\infty} \sigma^{2 z-1} e^{-\sigma^{2}-p / \sigma^{2}} F_{1}\left(m ; b,-\alpha \sigma^{2}\right) \mathrm{d} \sigma . \quad$ Therefore

$\Gamma_{p}(z ; m, b, \alpha) \Gamma_{p}(n ; m, b, \alpha)=4 \int_{0}^{\infty} \int_{0}^{\infty} \sigma^{2 m-1} \omega^{2 n-1} \sigma^{2 z-1} e^{-\sigma^{2}-p / \sigma^{2}} \times e^{-\omega^{2}-p / \omega^{2}}{ }_{1} F_{1}\left(m ; b ;-\alpha \sigma^{2}\right){ }_{1} F_{1}\left(m ; b ;-\alpha \omega^{2}\right) \mathrm{d} \sigma \mathrm{d} \omega$.

Letting $\sigma=\gamma \cos \theta, \omega=\gamma \sin \theta$ in (33) gives (31).

Remark 4. $\alpha=0$ in (33) gives the product of extended gamma function (see [13]).
Remark 5. By putting $p=0, \alpha=0$ in (33), one obtains the standard relation between gamma and beta function.

Theorem 4. For confluent hypergeometric gamma (CHG) function (12), one has the following recurrence relationship:

$$
(m-1) \Gamma_{p}(z ; m \cdot b, \alpha)=(m-b) \Gamma_{p}(z ; m-1, b, \alpha)+(b-1) \Gamma_{p}(z ; m-1, b-1, \alpha) .
$$

Proof. The confluent hypergeometric functions ${ }_{1} F_{1}(\xi-1 ; \tau ; z), \quad{ }_{1} F_{1}(\xi+1 ; \tau ; z), \quad{ }_{1} F_{1}(\xi ; \tau-1 ; z), \quad$ and ${ }_{1} F_{1}(\xi ; \tau+1 ; z)$ in which one parameter is increased or decreased by unity are said to be contiguous to ${ }_{1} F_{1}(\xi ; \tau ; z)$. The following relations exist between ${ }_{1} F_{1}$ and two of its contiguous functions [14].

$$
\begin{aligned}
\tau_{1} F_{1}(\xi: \tau ; z)= & \tau_{1} F_{1}(\xi-1 ; \tau ; z) \\
& +z_{1} F_{1}(\xi ; \tau+1 ; z), \\
(1+\xi-\tau)_{1} F_{1}(\xi ; \tau ; z)= & \xi_{1} F_{1}(\xi+1 ; \tau ; z) \\
& -(\tau-1)_{1} F_{1}(\xi ; \tau-1 ; z) .
\end{aligned}
$$

Substituting the function ${ }_{1} F_{1}$ appearing in (36) and (12) and replacing $\xi$ by $m, \tau$ by $b$, and $z$ by $-\alpha t$, one obtains (34).

Theorem 5. Let $z, \rho, \mu \in \mathbb{C}$. Then,

$$
(z ; m, b, \alpha, p)_{\rho+\mu}=(z)_{\rho}(z+\rho ; m, b, \alpha, p)_{\mu} .
$$

Proof. From (22)-(24), we have

$$
\begin{aligned}
(z ; m, b, \alpha, p)_{\rho+\mu} & =\frac{\Gamma_{p}(z+\rho+\mu ; m, b, \alpha)}{\Gamma_{(z)}} \\
& =\frac{\Gamma(z+\rho)}{\Gamma(z+\rho)} \frac{\Gamma_{p}(z+\rho+\mu ; m, b, \alpha)}{\Gamma(z)} \\
& =(z)_{\rho}(z+\rho ; m, b, \alpha, p) .
\end{aligned}
$$

Remark 6. From equation (37), one can write

$$
(z ; m, b, \alpha, p)_{r+n}=(z)_{r}(z+r ; m, b, \alpha, p)_{n}, \quad\left(z \in \mathbb{C}, r, n \in \mathbb{N}_{0}\right)
$$

Now, we can derive the following properties of CHP symbol $(z ; m, b, \alpha, p)_{n}$ through familiar properties of classical and extended Pochhammer symbols $(z)_{n}$ and $(z ; \kappa)_{n}$ (see, for instance, $[2,7,15]$ ).

Corollary 1. For $l, r, k, n \in \mathbb{N}_{0}$ and $R \in \in \mathbb{N}$, we can derive following properties: 


$$
\begin{aligned}
& (z ; m, b, \alpha, p)_{n+r+l}=(z)_{n}(z+n)_{r}(z+n+r ; m, b, \alpha, p)_{l} \text {, } \\
& (z ; m, b, \alpha, p)_{n-r+l}=\frac{(-1)^{r}(z)_{n}}{(1-z-n)_{r}}(z+n-r ; m, b, \alpha, p)_{l}, \\
& (z ; m, b, \alpha, p)_{2 r+l}=2^{2 r}\left(\frac{z}{2}\right)_{r}\left(\frac{z+1}{2}\right)_{r}(z+2 r ; m, b, \alpha)_{l}, \\
& (z ; m, b, \alpha, p)_{R r+l}=R^{R r}\left(\frac{z}{R}\right)_{r}\left(\frac{z+1}{R}\right)_{r} \ldots\left(\frac{z+R-1}{R}\right)_{r}(z+R r ; m, b, \alpha, p)_{l} \text {, } \\
& (z+n ; m, b, \alpha, p)_{n+l}=(z+n)_{n}(z+2 n ; m, b, \alpha, p)_{l} \\
& =\frac{(z)_{2 n}}{(z)_{n}}(z+2 n ; m, b, \alpha, p)_{l}, \\
& (z+r ; m, b, \alpha, p)_{n+l}=\frac{(z)_{n}(z+n)_{r}}{(z)_{r}}(z+n+r ; m, b, \alpha, p)_{l}, \\
& (z+k r ; m, b, \alpha, p)_{k n+l}=\frac{(z)_{k n+k r}}{(z)_{k r}}(z+k n+k r ; m, b, \alpha, p)_{l}, \\
& (z-n ; m, b, \alpha, p)_{n+l}=(-1)^{n}(1-z)_{n}(z ; m, b, \alpha, p)_{l} \text {, } \\
& (z-n ; m, b, \alpha, p)_{n+l}=\frac{(1-z)_{r}(z)_{n}}{(1-z-n)_{r}}(z+n-r ; m, b, \alpha, p)_{l}, \\
& (z-k r ; m, b, \alpha, p)_{k n+l}=(-1)^{k r}(z)_{k n-k r}(1-z)_{k r}(z+k n-k r ; m, b, \alpha, p)_{l} \text {, } \\
& (z+r ; m, b, \alpha, p)_{n-r+l}=\frac{(z)_{n}}{(z)_{r}}(z+n ; m, b, \alpha, p)_{l} \text {, } \\
& (z-r ; m, b, \alpha, p)_{n-r+l}=\frac{(-1)^{r}(1-z)_{r}(z)_{n}}{(1-z-n)_{2 r}}(z+n-2 r ; m, b, \alpha, p)_{l}, \\
& \text { and } \\
& (-z ; m, b, \alpha, p)_{n+l}=(-1)^{r}(z-n+1)_{n}(-z+n ; m, b, \alpha, p)_{l} \text {. }
\end{aligned}
$$

\section{A Generalized Hypergeometric \\ Gamma Function}

We define a GHG function ${ }_{r} F_{q}($.$) in the following form:$

$$
{ }_{r} F_{q}\left[\left(\eta_{1} ; m, b, \alpha, p\right) \eta_{2}, \ldots, \eta_{r} ; \beta_{1}^{\prime}, \ldots \beta_{q}^{\prime} ; z\right]=\sum_{n=0}^{\infty} \frac{\left(\eta_{1} ; m, b, \alpha, p\right)_{n}\left(\eta_{2}\right)_{n} \ldots\left(\eta_{r}\right)_{n}}{\left(\beta_{1}^{\prime}\right)_{n} \ldots\left(\beta_{q}^{\prime}\right)_{n}} \frac{z^{n}}{n !}
$$

where $\eta_{i} \in \mathbb{C}$ for $i=1,2, \ldots$ r, $\beta_{j}^{\prime} \in \mathbb{C}$ for $j=1,2, \ldots$, q, and $\beta_{j}^{\prime} \neq 0,-1,-2, \ldots$

New extensions of Gauss hypergeometric and confluent hypergeometric functions, respectively, are defined by

$$
\begin{gathered}
{ }_{2} F_{1}\left[\left(\eta_{1} ; m, b, \alpha, p\right), \beta^{\prime} ; \gamma ; z\right]=\sum_{n=0}^{\infty} \frac{\left(\eta_{1} ; m, b, \alpha, p\right)_{n}\left(\beta^{\prime}\right)_{n}}{(\gamma)_{n}} \frac{z^{n}}{n !}, \\
{ }_{1} F_{1}\left[\left(\eta_{1} ; m, b, \alpha, p\right) ; \gamma ; z\right]=\sum_{n=0}^{\infty} \frac{\left(\eta_{1} ; m, b, \alpha, p\right)_{n} \frac{z^{n}}{(\gamma)_{n}} .}{n !}
\end{gathered}
$$

Remark 7. Let us assume $m=b, \alpha=0$, and $p=\kappa$ in (42); one will get the following generalized hypergeometric function defined by Srivastava et al. [2]:

$$
\begin{aligned}
& { }_{r} F_{q}\left[\left(\eta_{1} ; \kappa\right), \quad \eta_{2}, \ldots, \delta_{r} ; \beta_{1}, \ldots \beta_{q} ; z\right] \\
& =\sum_{n=0}^{\infty} \frac{\left(\eta_{1} ; \kappa\right)_{n}\left(\eta_{2}\right)_{n} \ldots\left(\eta_{r}\right)_{n}}{\left(\beta_{1}\right)_{n} \ldots\left(\beta_{q}\right)_{n}} \frac{z^{n}}{n !},
\end{aligned}
$$

where $\eta_{i} \in \mathbb{C}$ for $i=1,2, \ldots, \mathrm{r}, \beta_{j}^{\prime} \in \mathbb{C}$ for $j=1,2, \ldots, \mathrm{q}$, and $\beta_{j}^{\prime} \neq 0,-1,-2, \ldots$. 
Remark 8. If we assume $\kappa=0$ in (44), we then obtain the classical generalized hypergeometric function [7]:

$$
{ }_{r} F_{q}\left[\left(\eta_{1}\right), \eta_{2}, \ldots, \eta_{r} ; \beta_{1}^{\prime}, \ldots \beta_{q} ; z\right]=\sum_{n=0}^{\infty} \frac{\left(\eta_{1}\right)_{n}\left(\eta_{2}\right)_{n} \ldots\left(\eta_{r}\right)_{n}}{\left(\beta_{1}^{\prime}\right)_{n} \ldots\left(\beta_{q}^{\prime}\right)_{n}},
$$

where $\eta_{i} \in \mathbb{C}$ for $i=1,2, \cdots r, \beta_{j}^{\prime} \in \mathbb{C}$ for $j=1,2, \ldots, q$, and $\beta_{j}^{\prime} \neq 0,-1,-2, \cdots$.

Now in the following theorems, different integral representations of GHG function (42) are derived.

Theorem 6. If $\eta_{i} \in \mathbb{C}$ for $i=1,2, \ldots, \beta_{j}^{\prime} \in \mathbb{C}$ for $j=1,2, \ldots$, $q$, and $\beta_{j}^{\prime} \neq 0,-1,-2, \ldots$, then

$$
{ }_{r} F_{q}\left[\left(\eta_{1} ; m, b, \alpha, p\right), \eta_{2}, \ldots, \eta_{r} ; \beta_{1}, \ldots \beta_{q} ; z\right]=\frac{1}{\Gamma\left(\eta_{1}\right)} \int_{0}^{\infty} t^{\eta_{1}+\rho-1} e^{-t-\frac{p}{t}}{ }_{1} F_{1}(m ; b,-\alpha t) \times_{r-1} F_{q}\left[\eta_{2}, \ldots, \eta_{r} ; \beta_{1}^{\prime}, \ldots \beta_{q}^{\prime} ; z t\right] .
$$

Proof. Applying integral representation of extended CHP symbol (26) in the definition of extended hypergeometric function (42), we have

$$
{ }_{r} F_{q}\left[\left(\eta_{1} ; m, b, \alpha, p\right), \eta_{2}, \ldots, \eta_{r} ; \beta_{1}^{\prime}, \ldots \beta_{q}^{\prime} ; z\right]=\frac{1}{\Gamma\left(\eta_{1}\right)} \sum_{n=0}^{\infty} \int_{0}^{\infty} t^{\eta_{1}+\rho-1} e^{-t-\frac{p}{t}} \times_{1} F_{1}(m ; b,-\alpha t) \frac{\left(\eta_{2}\right)_{n} \ldots\left(\delta_{r}\right)_{n}}{\left(\beta_{1}^{\prime}\right)_{n} \ldots\left(\beta_{q}^{\prime}\right)_{n}} \frac{z^{n}}{n !} \mathrm{d} t
$$

Exchanging integration and summation order and then by applying generalized hypergeometric function (45), we obtained the desired result.
Theorem 7. If $\mathfrak{R}(\gamma)>\mathfrak{R}\left(\beta^{\prime}\right)>0 ; \mathfrak{R}(m, b, \alpha) \geq 0$, then the beta-type integral associated with the GHG function is defined by

$$
\begin{aligned}
& { }_{r} F_{q}\left[\left(\eta_{1} ; m, b, \alpha, p\right), \eta_{2}, \ldots, \eta_{r-1}, \beta^{\prime} ; \beta_{1}^{\prime}, \ldots \beta_{q-1}^{\prime}, \gamma ; z\right] \\
& =\frac{1}{B\left(\beta^{\prime}, \gamma-\beta^{\prime}\right)} \int_{0}^{1} t^{\beta^{\prime}-1}(1-t)^{\gamma-\beta^{\prime}-1} \times_{r-1} F_{q-1}\left[\delta_{2}, \ldots, \delta_{r} ; \beta_{1}^{\prime}, \ldots \beta_{q-1}^{\prime} ; z t\right] \mathrm{d} t .
\end{aligned}
$$

Proof. From (42), we have

$$
\begin{aligned}
& \left.{ }_{r} F_{q}\left[\left(\eta_{1} ; m, b, \alpha, p\right)\right) \eta_{2}, \ldots, \delta_{r} ; \beta_{1}^{\prime}, \ldots, \beta_{q}^{\prime} ; z\right] \\
& =\sum_{n=0}^{\infty} \frac{\left(\eta_{1} ; m, b, \alpha, p\right)_{n}\left(\eta_{2}\right)_{n} \ldots\left(\eta_{r-1}\right)\left(\beta^{\prime}\right)_{n}}{\left(\eta_{1}^{\prime}\right)_{n} \ldots\left(\beta_{q-1}^{\prime}\right)_{n}(\gamma)_{n}} \frac{z^{n}}{n !} .
\end{aligned}
$$

We know that

$$
\begin{aligned}
\frac{\left(\beta^{\prime}\right)_{n}}{(\gamma)_{n}} & =\frac{B\left(\beta^{\prime}+n, \gamma-\beta^{\prime}\right)}{B\left(\beta^{\prime}, \gamma-\beta^{\prime}\right)} \\
& =\frac{1}{B\left(\beta^{\prime}, \gamma-\beta^{\prime}\right)} \int_{0}^{1} t^{\beta^{\prime}+n-1}(1-t)^{\gamma-\beta^{\prime}-1} \mathrm{~d} t .
\end{aligned}
$$

Combining (49) and (45) solves the theorem.

Theorem 8. If $\Re(m, b, \alpha) \geq 0, n \in \mathbb{N}_{0}$, then the $n^{\text {th }}$ order derivative of $G H G$ function (42) is given by

$$
\begin{aligned}
& \frac{\mathrm{d}^{n}}{\mathrm{~d} z^{n}}\left\{{ }_{r} F_{q}\left[\left(\eta_{1} ; m, b, \alpha, p\right), \eta_{2}, \ldots, \eta_{r} ; \beta_{1}^{\prime}, \ldots \beta_{q}^{\prime} ; z\right]\right\} \\
& =\frac{\left(\eta_{1}\right)_{n}\left(\eta_{2}\right)_{n} \ldots\left(\eta_{r}\right)_{n}}{\left(\beta_{1}^{\prime}\right)_{n} \ldots\left(\beta_{q}^{\prime}\right)_{n}} \\
& \quad \times{ }_{r} F_{q}\left[\left(\eta_{1}+n ; m, b, \alpha, p\right), \eta_{2}+n, \ldots, \eta_{r}+n ; \beta_{1}^{\prime}, \ldots \beta_{q}^{\prime} ; z\right] .
\end{aligned}
$$

Proof. The proof of (51) is minimal for $n \in 0$. Consider if $n \neq 0$, then

$$
\begin{aligned}
& \frac{\mathrm{d}}{\mathrm{d} z}\left\{{ }_{r} F_{q}\left[\left(\eta_{1} ; m, b, \alpha, p\right), \eta_{2}, \ldots, \eta_{r} ; \beta_{1}^{\prime}, \ldots \beta_{q}^{\prime} ; z\right]\right\} \\
& =\sum_{n=1}^{\infty} \frac{\left(\eta_{1} ; m, b, \alpha, p\right)_{n}\left(\eta_{2}\right)_{n} \ldots\left(\eta_{r}\right)_{n}}{\left(\beta_{1}^{\prime}\right)_{n} \ldots\left(\beta_{q}^{\prime}\right)_{n}} \frac{Z^{n-1}}{(n-1) !} \\
& =\sum_{n=0}^{\infty} \frac{\left(\eta_{1} ; m, b, \alpha, p\right)_{n+1}\left(\eta_{2}\right)_{n+1}, \ldots,\left(\eta_{r}\right)_{n+1}}{\left(\beta_{1}^{\prime}\right)_{n+1}, \ldots,\left(\beta_{q}^{\prime}\right)_{n+1}} \frac{Z^{n}}{n !} .
\end{aligned}
$$


In light of (37) and applying $(x)_{n+1}=x(x+1)_{n}$ in (54), it results in

$$
\begin{aligned}
& \frac{\mathrm{d}}{\mathrm{d} z}\left\{{ }_{r} F_{q}\left[\left(\eta_{1} ; m, b, \alpha, p\right), \eta_{2}, \ldots, \eta_{r} ; \beta_{1}^{\prime}, \ldots \beta_{q}^{\prime} ; z\right]\right\} \\
& \left.=\frac{\left(\eta_{1}\right)\left(\eta_{2}\right) \ldots\left(\eta_{r}\right)}{\left(\beta_{1}^{\prime}\right) \ldots\left(\beta_{q}^{\prime}\right)} F_{q}\left[\left(\eta_{1} ; m, b, \alpha, p\right), \eta_{2}+1, \ldots, \eta_{r}+1 ; \beta_{1}^{\prime}+1, \ldots \beta_{q}^{\prime}+1 ; z\right]\right\} .
\end{aligned}
$$

Differentiating $n$ times proves the theorem.

Corollary 2. If in (40), we consider the cases $r=q=1$ and $r=2, q=1$, then the nth derivative of the new extended confluent and Gauss hypergeometric functions become, respectively,

$$
\begin{aligned}
& \frac{\mathrm{d}^{n}}{\mathrm{~d} z^{n}}\left\{{ }_{1} F_{1}[(\eta ; m, b, \alpha, p) ; \gamma ; z]\right\}=\frac{(\eta)_{n}}{(\gamma)_{n}}{ }_{1} F_{1}[(\eta+n ; m, b, \alpha, p) ; \gamma+n ; z], \\
& \frac{\mathrm{d}^{n}}{\mathrm{~d} z^{n}}\left\{{ }_{2} F_{1}\left[(\eta ; m, b, \alpha, p) ; \beta^{\prime} ; \gamma ; z\right]=\frac{(\eta)_{n}\left(\beta^{\prime}\right)_{n}}{(\gamma)_{n}}\right. \\
& \quad \times{ }_{2} F_{1}\left[(\eta+n ; m, b, \alpha, p) ; \beta^{\prime}+n ; \gamma+n ; z\right] .
\end{aligned}
$$

\section{Families of Generating Function Relations}

Let $\Delta(R, z)$ indicate the below $\mathrm{R}$ parameters array.

$$
\frac{z}{R}, \frac{z}{R+1}, \ldots, \frac{z+R-1}{R},(z \in \mathbb{C} ; R \in \mathbb{N}) .
$$

If $R=0$, then the $\Delta(R, z)$ array will be null (see [16-18]).

Theorem 9. The following confluent hypergeometric gamma (CHG) generating function is defined by

$$
\begin{aligned}
& \sum_{n=0}^{\infty} \frac{(z)_{n}}{n !} r_{r+R} F_{q}\left[\begin{array}{c}
\Delta(R, z+n),\left(\eta_{1} ; m, b, \alpha, p\right), \eta_{2} \ldots, \eta_{r} ; \\
\beta_{1}^{\prime} \ldots, \beta_{q}^{\prime} ;
\end{array}\right] x^{n} \\
& =(1-x)^{-z}{ }_{r+R} F_{q}\left[\begin{array}{c}
\Delta(R, z),\left(\eta_{1} ; m, b, \alpha, p\right), \eta_{2} \ldots, \eta_{r} ; \\
\beta_{1}^{\prime} \ldots, \beta_{q}^{\prime} ;
\end{array} \frac{z}{(1-x)^{R}}\right],
\end{aligned}
$$

$(|x|<1, z \in \mathbb{C}, R \in \mathbb{N})$ 
provided every element of (56) exists.

Proof. Using the identity

$$
\sum_{n=0}^{\infty} \frac{(z)_{n}}{n !} z^{n}=(1-z)^{-z},(|z|<1 ; z \in \mathbb{C})
$$

to generalize hypergeometric function extension (GHF) (42) results in required assertion (56) of the Theorem 5 .

Theorem 10. The below generating function exists:

$$
\begin{aligned}
& \sum_{n=0}^{\infty} \frac{(z)_{n}}{n !}{ }^{r+R} F_{q}\left[\begin{array}{c}
\Delta(R,-n),\left(\eta_{1} ; m, b, \alpha, p\right), \eta_{2}, \ldots, \eta_{r} ; \\
\beta_{1}^{\prime} \ldots, \beta_{q}^{\prime}
\end{array}\right] x^{n} \\
& =(1-x)^{-z+R} F_{q}\left[\begin{array}{c}
\Delta(R, z),\left(\eta_{1} ; m, b, \alpha, p\right), z_{2} \ldots, \eta_{r} ; \\
\beta_{1}^{\prime} \ldots, \beta_{q}^{\prime} ;
\end{array}\right] \\
& \sum_{n=0}^{\infty} \frac{(z)_{n}}{n !}{ }^{r+2 R} F_{q}\left[\begin{array}{c}
\Delta(R,-n), \Delta(R, z+n),\left(\eta_{1} ; m, b, \alpha, p\right), \eta_{2} \ldots, \eta_{r} ; z \\
\beta_{1}^{\prime} \ldots, \beta_{q}^{\prime}
\end{array}\right] x^{n}
\end{aligned}
$$

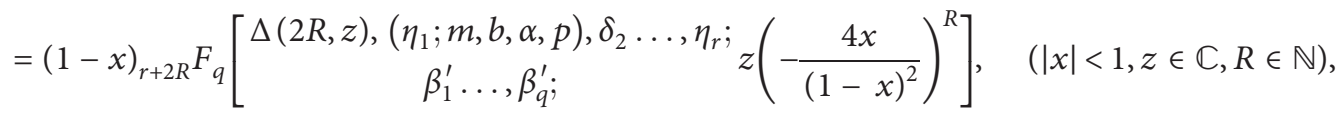

and

$$
\begin{aligned}
& \sum_{n=0}^{\infty} \frac{(z)_{n}}{n !}{ }_{r+R} F_{q+R}\left[\begin{array}{c}
\Delta(R,-n),\left(\eta_{1} ; m, b, \alpha, p\right), \eta_{2} \ldots, \eta_{r} ; \\
\beta_{1}^{\prime} \ldots, \beta_{q}^{\prime} ;
\end{array}\right] x^{n} \\
& =(1-x)^{-z}{ }_{r+2 R} F_{R}\left[\begin{array}{c}
\Delta(2 R, z),\left(\delta_{1} ; m, b, \alpha, p\right), \delta_{2} \ldots, \delta_{r} ; \\
\Delta(R, 1-z-n), \beta_{1}^{\prime} \ldots, \beta_{q}^{\prime} ;
\end{array}\right], \quad(|x|<1, z \in \mathbb{C}, R \in \mathbb{N}),
\end{aligned}
$$

provided every element of (58)-(60) exists.

Proof. The proof of (58)-(60) is similar to that of Theorem 6.

$$
\begin{aligned}
& \sum_{n=0}^{\infty} \frac{(z)_{n}}{n !}{ }_{r+1} F_{q}\left[\begin{array}{c}
(z+n),\left(\eta_{1} ; m, b, \alpha, p\right), \eta_{2} \ldots, \eta_{r} ; \\
\beta_{1}^{\prime} \ldots, \beta_{q}^{\prime} ;
\end{array}\right] x^{n} \\
& =(1-x)_{r+1}^{-z} F_{q}\left[\begin{array}{c}
z,\left(\eta_{1} ; m, b, \alpha, p\right), \eta_{2} \cdots, \eta_{r} ; \\
\beta_{1}^{\prime} \ldots, \beta_{q}^{\prime} ;
\end{array}\right], \quad(|x|<1, z \in \mathbb{C}), \\
& \sum_{n=0}^{\infty} \frac{(z)_{n}}{n !}{ }_{r+1} F_{q}\left[\begin{array}{c}
-n,\left(\eta_{1} ; m, b, \alpha, p\right), \eta_{2} \ldots, \eta_{r} ; z \\
\beta_{1}^{\prime} \ldots, \beta_{q}^{\prime} ;
\end{array}\right] x^{n} \\
& =(1-x)_{r+1}^{-z} F_{q}\left[\begin{array}{c}
z,\left(\left(\eta_{1} ; m, b, \alpha, p\right), \eta_{2} \ldots, \eta_{r} ; z\left(-\frac{x}{(1-x)}\right)\right. \\
\beta_{1}^{\prime} \ldots, \beta_{q}^{\prime} ;
\end{array}\right. \text {, } \\
& (|x|<1, z \in \mathbb{C}) \text {, }
\end{aligned}
$$
exist. 


$$
\begin{aligned}
& \sum_{n=0}^{\infty} \frac{(z)_{n}}{n !}{ }_{r+2} F_{q}\left[\begin{array}{c}
-n,\left(\eta_{1} ; m, b, \alpha, p\right), \eta_{2} \ldots, \eta_{r} ; z \\
\beta_{1}^{\prime} \ldots, \beta_{q}^{\prime} ;
\end{array}\right] x^{n} \\
& =(1-x)^{-z+2} F_{q}\left[\begin{array}{c}
\left.\Delta(2, z),\left(\eta_{1} ; m, b, \alpha, p\right), \eta_{2} \ldots, \eta_{r} ; z\left(-\frac{4 x}{(1-x)^{2}}\right)\right], \\
\beta_{1}^{\prime} \ldots, \beta_{q}^{\prime} ;
\end{array}\right. \\
& (|x|<1, z \in \mathbb{C}, N \in \mathbb{N}),
\end{aligned}
$$

and

$$
\begin{aligned}
& \sum_{n=0}^{\infty} \frac{(z)_{n}}{n !}{ }_{r+1} F_{q+1}\left[\begin{array}{c}
-n,\left(\eta_{1} ; m, b, \alpha, p\right), \eta_{2} \ldots, \eta_{r} ; \\
1-z-n, \beta_{1}^{\prime} \ldots, \beta_{q}^{\prime} ;
\end{array}\right] x^{n} \\
& =(1-x)^{-z}{ }_{r} F_{q}\left[\begin{array}{c}
\Delta(2, z),\left(\eta_{1} ; m, b, \alpha, p\right), \eta_{2} \ldots, \eta_{r} ; \\
\beta_{1}^{\prime} \ldots, \beta_{q}^{\prime} ;
\end{array}\right],
\end{aligned}
$$$$
(|x|<1, z \in \mathbb{C})
$$

provided that each number of (61)-(64) exists.

Proof. Assuming $R=1$ in Theorems 9 and 10, respectively, results in required assertions (61)-(64) of Corollary 3.

\section{Numerical Comparisons}

In Table 1, we compared classical gamma, extended gamma (3), and CHG (12) functions for different numerical values of $z$ in Computational Package Mathematica. We fixed values of $m=1.9, b=2.3, \alpha=1.1, p=2.1$. For different numerical values of $z$, extended gamma function (3) gives lesser values than gamma function while CHG (12) gives the least values. We also compared integral and closed forms of $\mathrm{CHG}$ functions in Table 2. We observed that results for both integral and closed forms are the same, but for the closed form, numerical values are obtained more quickly compared to the integral form.

\section{Conclusion}

In this paper, further generalization of the extended gamma function of Chaudhry and Zubair [12] is introduced. An associated confluent hypergeometric Pochhammer (CHP) symbol is also defined. Using confluent hypergeometric gamma (CHG) function, further generalizations of hypergeometric functions are also discussed. The new extended gamma function involving confluent hypergeometric was found to be useful in a variety of heat conduction problems. Additionally, we introduced a few families of generating functions for generalized hypergeometric function. Some special cases are also derived. We may develop new extensions of various special functions by applying confluent hypergeometric Pochhammer (CHP) symbol as shown in (24).

\section{Data Availability}

No data were used to support this study.

\section{Conflicts of Interest}

The authors declare that they have no conflicts of interest.

\section{Acknowledgments}

Thabet Abdeljawad would like to thank Prince Sultan University for supporting this study through research group Nonlinear Analysis Methods in Applied Mathematics (NAMAM) (group no. RG-DES-2017-01-17).

\section{References}

[1] M. A. Chaudhry and S. M. Zubair, On a Class of Incomplete Gamma Functions with Applications, CRC Press, Cleverland, OH, USA, 2001.

[2] H. M. Srivastava, A. Çetinkaya, and İ. Onur Kıymaz, "A certain generalized pochhammer symbol and its applications to hypergeometric functions," Applied Mathematics and Computation, vol. 226, pp. 484-491, 2014.

[3] H. M. Srivastava, G. Rahman, and K. S. Nisar, "Some extensions of the pochhammer symbol and the associated hypergeometric functions," Iranian Journal of Science and Technology, Transactions A: Science, vol. 43, no. 5, pp. 26012606, 2019.

[4] M. Safdar, G. Rahman, Z. Ullah, A. Ghaffar, and K. S. Nisar, "A new extension of the pochhammer symbol and its application to hypergeometric functions," International Journal of Applied and Computational Mathematics, vol. 5, p. 151, 2019.

[5] H. M. Srivastava, M. A. Chaudhry, and R. P. Agarwal, "The incomplete Pochhammer symbols and their applications to hypergeometric and related functions," Integral Transforms and Special Functions, vol. 23, no. 9, pp. 659-683, 2012.

[6] R. Şahin and O. Yağcl, "A new generalization of pochhammer symbol and its applications," Applied Mathematics and Nonlinear Sciences, vol. 5, pp. 255-266, 2020.

[7] E. D. Rainville, Special Functions, Vol. 211, Bronz, New York, NY, USA, 1960.

[8] S. B. Provost, A. Saboor, and M. Ahmad, "The gamma-weibull distribution,” Pak. J. Statist.vol. 27, pp. 111-131, 2011.

[9] I. Gradshteyn and I. Ryzhik's, A. Jeffrey, Tables of Integrals, Series, and Products, Academic Press, New York, NY, USA, 2014.

[10] M. Abramowitz and I. A. Stegun, Handbook of Mathematical Functions with Formulas, Graphs, and Mathematical Tables, New York, NY, USA.

[11] A. Mathai, A Handbook of Generalized Special Functions for Statistical and Physical Sciences, Oxford University Press, Oxford, USA. 
[12] M. A. Chaudhry and S. M. Zubair, "Generalized incomplete gamma functions with applications," Journal of Computational and Applied Mathematics, vol. 55, no. 99-123, 1994.

[13] M. A. Chaudhry and S. M. Zubair, "Extended gamma and digamma functions," Fractional Calculus and Applied Analysis, vol. 4, pp. 303-326, 2018.

[14] L. J. Slater, Confluent Hypergeometric Functions, Cambridge University Press, Cambridge, UK.

[15] A. P. Prudnikov, Y. A. Brychkov, and O. I Marichev, Integrals and series: special functions, CRC Press, London, UK, 1986.

[16] G. E. Andrews, R. Askey, and R. Roy, Special Functions. Encyclopedia of Mathematics and its Applications, Cambridge University Press, Cambridge, UK, 1999.

[17] Erdélyi, Higher Transcendental Functions, Vol. 1, McGrawHill, New York, NY, USA, 1953.

[18] H. M. Srivastava and H. L. Manocha, A Treatise on Generating Functions, Wiley, New York, NY, USA. 